ORIGINAL ARTICLE

\title{
Role of mesangial fibrinogen deposition in the pathogenesis of crescentic Henoch-Schönlein nephritis in children
}

\author{
J I Shin, J M Park, Y H Shin, J S Lee, H J Jeong
}

J Clin Pathol 2005;58:1147-1151. doi: 10.1136/jicp.2005.027409

See end of article for authors' affiliations

aurs affiliations

Correspondence to:

Professor J S Lee, 134

Shinchon-Dong,

Seodaemun-Ku, 120-752,

CPO Box 8044,

Department of Paediatrics,

Yonsei University College

of Medicine, Seoul, Korea;

jsyonse@yumc.yonsei.ac.

Accepted for publication 29 March 2005
Aims: To clarify the role of mesangial fibrinogen deposition in crescentic Henoch-Schönlein nephritis (HSN).

Methods: A retrospective analysis of 21 children with HSN treated with immunosuppressants. Serial renal biopsies were performed before and after treatment. They were divided into two groups according to the immunofluorescent course of fibrinogen deposition: group I $(n=9)$, no or decreased deposition; group II $(n=12)$, persistent or increased deposition.

Results: There were no differences between the two groups in renal manifestations or laboratory and histological findings at presentation. However, the activity index after immunosuppressive treatment was significantly decreased in group I (mean, 7.9 (SEM, 0.7) $v 2.9(0.4) ; p=0.008$ ) and unchanged in group II (mean, $6.8(S E M, 0.3) \vee 6.0(2.1))$. The chronicity index was unchanged in group I, but increased in group II (mean, 0.8 (SEM, 0.3) $v 1.8(0.3) ; p=0.02)$. Univariate analysis revealed that the only factor significantly related to persistent or increased fibrinogen deposition was age more than 9 years $(p=0.03$ ). Furthermore, the intensity of fibrinogen deposition at the second biopsy correlated positively with the age at onset $\left(R^{2}=0.306 ; p=0.009\right)$ and changes in the percentage of crescents (post-treatment crescents $(\%)$ minus pretreatment crescents (\%)) correlated positively with the intensity of fibrinogen deposition at the second biopsy $\left(R^{2}=0.193 ; p=0.046\right)$.

Conclusions: This study indicates that fibrinogen deposition has an important role to play in renal injury of crescentic HSN and reflects persistent severe histological activity.
$\mathrm{H}$ enoch-Schönlein purpura is a vasculitis with $\operatorname{IgA}$ dominant immune deposits that predominantly affect the skin, gastrointestinal tract, joints, and kidneys. Although Henoch-Schönlein purpura is usually acute and self limited, the longterm prognosis is dependent on the severity of renal involvement (Henoch-Schönlein nephritis; HSN). Patients with nephrotic range proteinuria, nephrotic/nephritic syndrome at onset, and a high percentage of crescents show a progressive course. ${ }^{2}{ }^{3}$ In specialised nephrology centres, an estimated $10 \%$ or more of children with HSN progress to end stage renal failure..$^{2-4}$

"It was suggested that fibrinolytic treatment should be dependent on the characteristics of the individual patient because crescents associated with Henoch-Schönlein nephritis are not always fibrinogen dependent"

Previous studies reported that several immunosuppressive agents have a beneficial effect on HSN ${ }^{4-6}$ Recently, Watanabe et al and Kawasaki et al described the efficacy of urokinase in the treatment of severe HSN, and suggested that fibrinolytic treatment could help relieve an intraglomerular hypercoagulable state related to renal injury and preserve renal function in patients with severe crescentic HSN. ${ }^{7-9}$ However, it was suggested that fibrinolytic treatment should be dependent on the characteristics of the individual patient because crescents associated with HSN are not always fibrinogen dependent.

Therefore, we compared the clinical and histological findings between patients with no or decreased fibrinogen deposits and those with persistent or increased deposits by serial renal biopsy to identify the role of fibrinogen deposition in crescentic HSN.

\section{PATIENTS AND METHODS \\ Patients}

From the years 1990 to 2002 we analysed the serial renal biopsy specimens of 21 (15 male and six female) children with at least grade IIIa HSN (see later), aged 3.6-15.5 years. HSN was diagnosed when haematuria and/or proteinuria were associated with a characteristic purpuric eruption and/ or abdominal or joint pain, and the presence of glomerular IgA deposits was the main diagnostic criterion. Patients with IgA nephropathy without a classic Henoch-Schönlein purpura rash were excluded. All patients were treated with prednisolone and immunosuppressants (azathioprine or cyclosporine). They were divided into two groups according to the immunofluorescent (IF) course of fibrinogen deposition: group I ( $\mathrm{n}=9$ ), no deposition or decreased deposition; group II $(n=12)$, persistent or increased deposition. The mean duration of follow up was 5.8 years (range, 1-12).

The clinical outcome at the end of immunosuppressive treatment or at the latest observation was graded as follows ${ }^{3}$ : (A) normal urine and renal function; (B) microscopic haematuria or proteinuria less than $40 \mathrm{mg} / \mathrm{m}^{2} /$ hour; (C) active renal disease with proteinuria of $40 \mathrm{mg} / \mathrm{m}^{2} /$ hour and a glomerular filtration rate of $60 \mathrm{ml} / \mathrm{min} / 1.73 \mathrm{~m}^{2}$ or greater; (D) renal insufficiency. Our study was approved by the institutional review board and the research ethics committee of Yonsei Severance Hospital, Korea.

\section{Histopathology}

All patients underwent percutaneous renal biopsy before and after immunosuppressive treatment. The mean interval between biopsies was 11.2 months (range, 8-14), and was

Abbreviations: IF, immunofluorescent; ISKDC, International Study of Kidney Disease in Children; HSN, Henoch-Schönlein nephritis 
Table 1 Clinical characteristics of the two groups

\begin{tabular}{|c|c|c|}
\hline & Group I $(n=9)$ & Group II $(n=12)$ \\
\hline Age at onset (years)* & $7.4(1.3)$ & $10.2(0.9)$ \\
\hline Age $>9$ years $\dagger$ & 2 & 9 \\
\hline Sex (male:female) & $6: 3$ & $9: 3$ \\
\hline Follow up (years) & $5.2(0.8)$ & $6.3(0.9)$ \\
\hline \multicolumn{3}{|l|}{ Initial renal manifestations } \\
\hline Macroscopic haematuria & 2 & 5 \\
\hline Hypertension & 2 & 3 \\
\hline Mild proteinuria & 1 & 2 \\
\hline Nephrotic range proteinuria & 6 & 7 \\
\hline Nephrotic syndrome & 2 & 3 \\
\hline Acute nephritic pictures & 1 & 1 \\
\hline \multicolumn{3}{|l|}{ Treatment } \\
\hline Azathioprine & 4 & 5 \\
\hline Cyclosporine & 5 & 7 \\
\hline \multicolumn{3}{|l|}{ Clinical outcome (A/B/C/D) } \\
\hline At the end of treatment & $4 / 5 / 0 / 0$ & $5 / 5 / 2 / 0$ \\
\hline At the latest observation & $7 / 2 / 0 / 0$ & $8 / 3 / 0 / 1$ \\
\hline
\end{tabular}

similar between the two groups. The glomerular changes were graded according to the classification of the International Study of Kidney Disease in Children (ISKDC) (grade I, minimal alterations; grade II, mesangial proliferation; grade IIIa/b, focal/diffuse proliferation or sclerosis with $<50 \%$ crescents; grade IVa/b, focal/diffuse proliferation or sclerosis with $50-75 \%$ crescents; grade $\mathrm{Va} / \mathrm{b}$, focal/diffuse proliferation or sclerosis with $>75 \%$ crescents; grade VI, membranoproliferative glomerulonephritis). ${ }^{2}$ Renal biopsy specimens were also scored semiquantitatively using the modified scoring system of Foster et al. ${ }^{5}$ Acute changes included mesangial hypercellularity $(0-3)$, mesangial matrix increase (0-3), endothelial swelling (0-2), interstitial mononuclear infiltrate $(0-2)$, interstitial oedema $(0-2)$, tubular damage (0-2), cellular crescents $(0-3)$, basement membrane adhesion to Bowman's capsule (0-3), glomerular neutrophils (0-3), and fibrinoid necrosis (0-3). Chronic renal injury was estimated by interstitial fibrosis and tubular atrophy (0-2), fibrous crescents (0-3), global sclerosis $(0-3)$, and vascular hyalinosis and intimal hyperplasia $(0-1)$. The sum of these numbers comprised the activity index and the chronicity index, respectively. The scores from four factors (interstitial mononuclear infiltrate, interstitial oedema, tubular damage, and interstitial fibrosis and tubular atrophy) were combined into the tubulointerstitial scores.

For the IF microscopic examination, a portion of the fresh renal tissue was frozen and exposed to fluorescein isothiocyanate conjugated antihuman IgG, IgA, IgM, C3, and fibrinogen. The overall intensity of IF was also scored semiquantitatively, as follows: 0 , negative; 0.5 , trace; 1 , mild; 2 , moderate; and 3 , severe.

\section{Statistical analysis}

Statistical analysis was performed using SPSS version 11.0 (SPSS Inc, Chicago, Illinois, USA). Continuous variables were expressed as mean values (SEM). Variables were compared using the Mann-Whitney test and Fisher's exact test. Differences between biopsy findings before and after treatment were assessed by the Wilcoxon signed rank test. Correlation between two variables was assessed by Spearman's rank correlation test; $\mathrm{p}<0.05$ was considered significant.

\section{RESULTS}

\section{Patients' characteristics}

There were no differences between the two groups with regard to sex, hypertension, macroscopic haematuria, nephrotic/nephritic syndrome, or treatment. The duration of follow up was similar for the two groups. The age at onset had borderline significance $(\mathrm{p}=0.058)$, but data analysis at various cutoff values of age revealed that an age more than 9 years was a significant factor related to persistent or increased fibrinogen deposition $(\mathrm{p}=0.03)$.

Twenty of the 21 patients showed normal urinalysis or minor urinary abnormalities at the latest observation. Although one patient in group II progressed to chronic renal insufficiency, there was no significant difference between the two groups in the clinical outcome at the end of immunosuppressive treatment or at the latest observation (table 1).

\section{Laboratory findings}

There were no significant differences between the two groups in urinary protein excretion, serum albumin, creatinine, and creatinine clearance at the first and second biopsy. In both groups, urinary protein excretion was decreased significantly and serum albumin was increased after immunosuppressive treatment, whereas serum creatinine and creatinine clearance did not change (table 2 ).

\section{Histopathological findings}

Table 3 shows the light microscopic findings of the 21 patients. At the first renal biopsy, the histological grade of the ISKDC and the activity indices did not differ between the two groups. The percentage of crescents in group I was higher than that in group II $(p=0.025)$. The second biopsy from patients in group I showed improvement $(n=9)$ of the histological grade of the ISKDC $(p=0.004)$ and a decrease in the activity index ( $p=0.008)$, whereas the second biopsy from patients in group II showed improvement $(n=2)$, no changes $(n=7)$, or progression $(n=3)$ of the grade of the ISKDC and persistent increase of the activity index. The percentage of crescents at the second biopsy was decreased in group I, but unchanged in group II. There were no differences between the two groups with regard to the chronicity index and tubulointerstitial scores of the first renal biopsy. At the second biopsy, however, the chronicity index $(p=0.012)$ and tubulointerstitial scores $(p=0.009)$ in group II were significantly higher than those in group I.

Table 4 shows the IF results. We used the IF course of mesangial fibrinogen deposition as the criterion for

Table 2 Laboratory findings in the two groups

\begin{tabular}{|c|c|c|c|c|}
\hline \multirow[b]{2}{*}{ Variables } & \multicolumn{2}{|l|}{ First biopsy } & \multicolumn{2}{|l|}{ Second biopsy } \\
\hline & Group I (n=9) & Group II $(n=12)$ & Group I (n=9) & Group II $(n=12)$ \\
\hline $\begin{array}{l}\text { Proteinuria }\left(\mathrm{g} / \mathrm{m}^{2} / \text { day) }\right. \\
\text { Albumin }(\mathrm{g} / \mathrm{l}) \\
\text { Creatinine }(\mathrm{mg} / \mathrm{l}) \\
\mathrm{CCr}\left(\mathrm{ml} / \mathrm{min} / 1.73 \mathrm{~m}^{2}\right)\end{array}$ & $\begin{array}{l}4.6(1.4) \\
30(2.0) \\
6.0(1.0) \\
82.0(6.1)\end{array}$ & $\begin{array}{l}5.4(1.3) \\
32(3.0) \\
7.0(1.0) \\
109.0(10.2)\end{array}$ & $\begin{array}{l}0.1(0.03)^{*} \\
44(1.0)^{*} \\
5.0(1.0) \\
117.0(22.1)\end{array}$ & $\begin{array}{l}0.5(0.1)^{*} \\
42(1.0)^{*} \\
7.0(1.0) \\
104.9(7.7)\end{array}$ \\
\hline
\end{tabular}


Table 3 Light microscopic findings in the two groups

\begin{tabular}{|c|c|c|c|c|c|c|}
\hline \multirow[b]{2}{*}{ Variables } & \multicolumn{2}{|l|}{ First biopsy } & \multirow[b]{2}{*}{ p Value } & \multicolumn{2}{|l|}{ Second biopsy } & \multirow[b]{2}{*}{ p Value } \\
\hline & Group I $(n=9)$ & Group II $(n=12)$ & & Group I ( $n=9$ ) & Group II $(n=12)$ & \\
\hline ISKDC & & & NS & & & 0.015 \\
\hline 1 & 0 & 0 & & 2 & 0 & \\
\hline$\|$ & 0 & 0 & & 6 & 2 & \\
\hline Illa & 6 & 9 & & 1 & 6 & \\
\hline Illb & 2 & 3 & & 0 & 3 & \\
\hline $\mathrm{IVb}$ & $\overline{1}$ & 0 & & 0 & 1 & \\
\hline $\mathrm{Al}$ & $7.9(0.7)$ & $6.8(0.3)$ & NS & $2.9(0.4)^{*}$ & $6.0(2.1)$ & 0.001 \\
\hline $\mathrm{Cl}$ & $0.4(0.2)$ & $0.8(0.3)$ & NS & $0.4(0.2)$ & $1.8(0.3) \dagger$ & 0.012 \\
\hline TI score & $1.0(0.5)$ & $1.7(0.4)$ & NS & $0.4(0.2)$ & $3.1(0.8)$ & 0.009 \\
\hline Crescents (\%) & $21.9(5.8)$ & $12.4(3.3)$ & NS & $1.4(1.4)^{*}$ & $13.9(5.0)$ & 0.019 \\
\hline
\end{tabular}

separating groups I and II. At the first biopsy, the amount of IgG, IgA, IgM, C3, and fibrinogen in the deposits did not differ between the two groups. At the second biopsy, however, the degree of mesangial fibrinogen deposition in group I was decreased compared with the first biopsy (mean, 1.4 (SEM, 0.5) v $0.4(0.2) ; \mathrm{p}=0.031)$, whereas it was slightly increased in group II (mean, 0.8 (SEM, 0.2) $v 1.1$ $(0.1) ; p=0.125)$, although it was not significantly different. Thus, mesangial fibrinogen deposits at the second biopsy in group II were significantly higher than those in group I (mean, $0.4(\mathrm{SD}, 0.2) v 1.1(0.1) ; \mathrm{p}=0.012)$. Other deposits did not differ between the two groups.

\section{Correlation of fibrinogen deposition with the age at onset and changes in the percentage of crescents}

The degree of mesangial fibrinogen deposition at the second biopsy correlated positively with the age at onset (fibrinogen deposition $=0.088 \times$ age at onset; $\left.R^{2}=0.306 ; \mathrm{p}=0.009\right)$. Changes in the percentage of crescents (post-treatment crescents (\%) - pretreatment crescents (\%); $\Delta$ crescents) correlated positively with the intensity of mesangial fibrinogen deposits at the second biopsy $(\Delta$ crescents $=12.368 \times$ fibrinogen deposition $\left.-17.623 ; R^{2}=0.193 ; \mathrm{p}=0.046\right)$ (fig 1).

\section{DISCUSSION}

Irrespective of aetiology, crescentic glomerulonephritis is indicative of severe glomerular injury, and the proportion of crescents is an important factor in determining prognosis. ${ }^{10}$ Crescent formation is associated with various factors, such as the accumulation of $\mathrm{T}$ cells, macrophages, tissue factor, components of delayed-type hypersensitivity, and fibrin deposition. ${ }^{11}$ Among these factors, several human and experimental studies have shown that glomerular fibrin deposition plays an important role in the mechanism of crescent formation. ${ }^{12}{ }^{13}$ In experimental models, defibrination decreased the number of crescents and the severity of renal insufficiency. ${ }^{14}{ }^{15}$ Recent studies, using genetic engineering, have shown that crescentic glomerulonephritis is reduced in mice that are genetically deficient in fibrinogen and increased in those overexpressing the plasminogen activator inhibitor- 1 gene. ${ }^{16} 17$

However, the mechanisms responsible for fibrin deposition and crescent formation and the precise role of coagulation in the pathogenesis of IgA nephropathy or HSN are still poorly understood. ${ }^{18-20}$ It was suggested that the activation of intraglomerular coagulation is one of the exacerbative factors in IgA nephropathy and HSN. ${ }^{18}{ }^{19}$ Miura et al reported that glomerular injury, such as glomerular adhesion to Bowman's capsule and crescents, was seen predominantly in glomeruli with $\alpha 2$-plasmin inhibitor and/or fibrinogen deposits in patients with IgA nephropathy, and suggested that the deposition of $\alpha 2$-plasmin inhibitor in vivo might lead to the accumulation of glomerular fibrinogen deposits. ${ }^{18}$ Ono et al reported that crosslinked fibrin in the glomeruli is associated with relatively low intraglomerular plasmin activity and intraglomerular coagulation, both in IgA nephropathy and in HSN. ${ }^{19}$

Therefore, it was thought that fibrinolytic treatment might help to preserve renal function in patients with IgA nephropathy or HSN. Several reports from the 1980s showed the beneficial effects of urokinase on severe IgA nephropathy, ${ }^{21}$ and Watanabe et al first used this treatment in a small number of patients with severe HSN. ${ }^{7}$ Recently, Kawasaki et al reported the efficacy of urokinase combined with a methylprednisolone pulse or cyclophosphamide in patients with severe crescentic HSN, ${ }^{89}$ because glomerular fibrinogen deposits are seen much more frequently in HSN than in IgA nephropathy. ${ }^{22}$

We postulated that there would be differences in crescent formation and glomerular fibrinogen deposition among patients with HSN, and that fibrinolytic treatment should be tailored to the characteristics of the individual patient because HSN is a heterogeneous disease with several histopathological presentations, and crescents associated with HSN are not always fibrinogen dependent. In our study,

Table 4 Immunofluorescent findings in the two groups

\begin{tabular}{|c|c|c|c|c|c|c|}
\hline \multirow[b]{2}{*}{ Variable } & \multicolumn{2}{|c|}{ First biopsy } & \multirow[b]{2}{*}{ p Value } & \multicolumn{2}{|c|}{ Second biopsy } & \multirow[b]{2}{*}{ p Value } \\
\hline & Group I & Group II & & Group I & Group II & \\
\hline $\lg G$ & $0.4(0.2)$ & $0.3(0.2)$ & NS & $0.2(0.1)$ & $0.1(0.1)$ & NS \\
\hline $\lg A$ & $1.9(0.4)$ & $1.9(0.2)$ & NS & $1.1(0.2)^{*}$ & $1.6(0.2)$ & NS \\
\hline $\lg M$ & $0.3(0.2)$ & $0.3(0.1)$ & NS & $0.3(0.1)$ & $0.2(0.1)$ & NS \\
\hline $\mathrm{C} 3$ & $0.6(0.3)$ & $0.5(0.1)$ & NS & $0.3(0.2)$ & $0.8(0.3)$ & NS \\
\hline Fibrinogen & $1.4(0.5)$ & $0.8(0.2)$ & NS & $0.4(0.2)^{*}$ & $1.1(0.1)$ & 0.012 \\
\hline
\end{tabular}

Values are mean (SEM).

${ }^{*} p<0.05$ when compared with the first biopsy. 

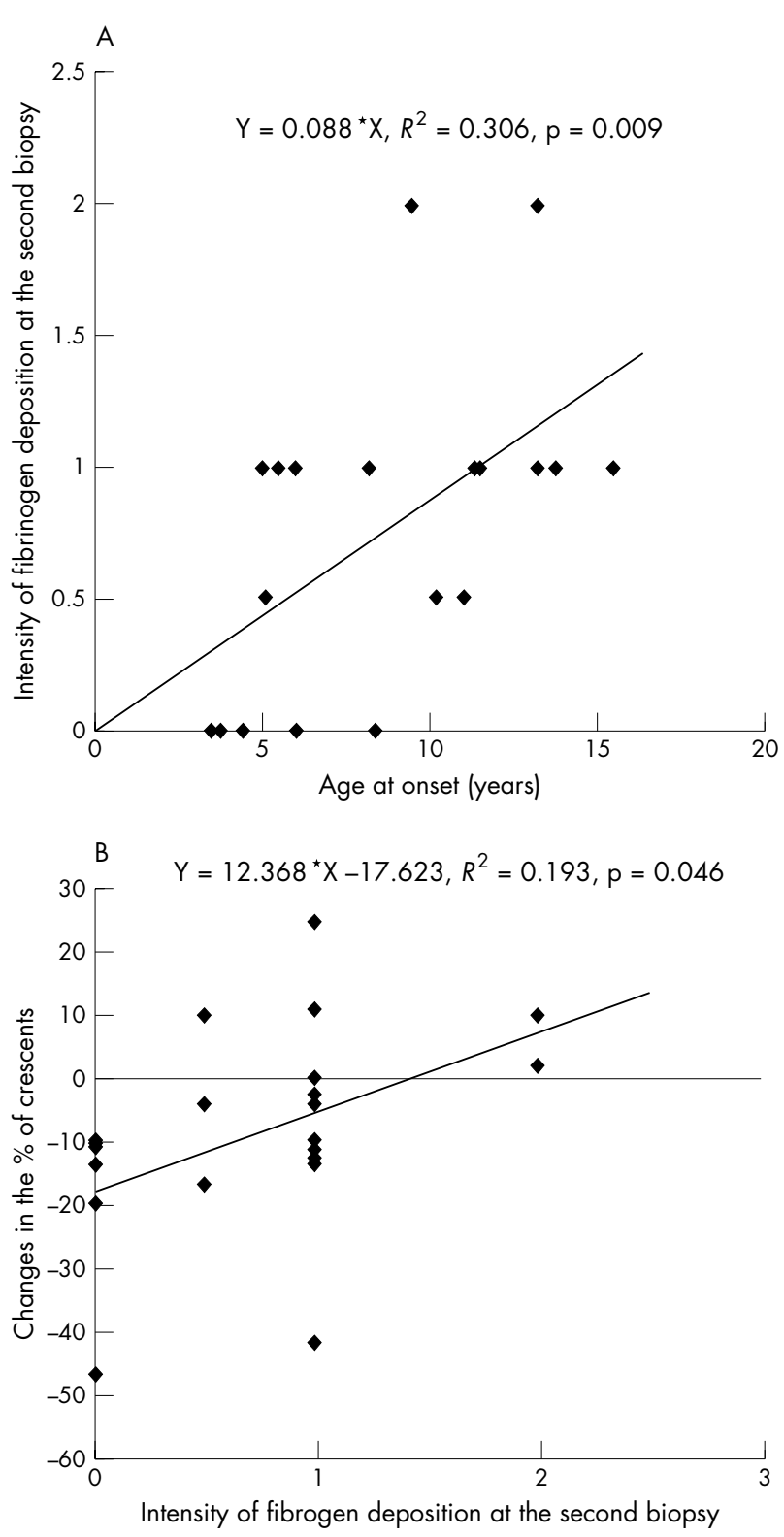

Figure 1 Correlations between the intensity of fibrinogen deposition at the second biopsy, age at onset, and change in the \% of crescents. (A) The intensity of fibrinogen deposition at the second biopsy correlated positively with the age at onset. (B) Change in the \% of crescents correlated positively with intensity of fibrinogen deposition at the second biopsy.

we classified the patients with HSN and crescentic change into two groups. This was not based on initial biopsy findings but on the subsequent course of the mesangial fibrinogen deposits. Although other authors had treated patients according to the fibrinogen deposits at the first biopsy, we found heterogeneous serial changes of fibrinogen deposition between the first and second biopsy: two patients had no fibrinogen deposits throughout the course of the disease and seven showed a reduction or disappearance of crescents and fibrinogen deposits with only immunosuppressants in group I, whereas three patients who had shown no fibrinogen deposits at the first biopsy developed new fibrinogen deposits at the second biopsy, and nine had persistent or increased fibrinogen deposits despite immunosuppressants in group II. Although clinical improvement was achieved even in patients with persistent or increased fibrinogen deposits, severe histological abnormalities persisted without regression in 10 of the 12 group II patients, suggesting that persistent or increased deposition of intraglomerular fibrinogen plays an important role in the pathogenesis of crescentic HSN.

Therefore, we were interested in discriminating between these two groups, and compared the clinical and histopathological findings at the time of the first biopsy. We found that an age greater than 9 years at onset was the only factor that was significantly related to persistent or increased fibrinogen deposition, and that age at onset was positively correlated with the intensity of fibrinogen deposition at the second biopsy. Although Tanaka et al reported that the age at onset in childhood IgA nephropathy has an effect on the histological changes seen after treatment with prednisolone, with children older than 10 showing more severe histological findings at the second biopsy, ${ }^{23}$ there has been no study describing the effect of age on histological responses to immunosuppressive treatment in severe HSN. We found that children with HSN greater than 9 years of age at onset had more severe fibrinogen deposits during the course of the disease and a worse response to immunosuppressants than those less than 9 years of age, suggesting that the age at onset influences the subsequent course of fibrinogen deposition and outcome. In addition, the intensity of fibrinogen deposition at the second biopsy positively correlated with the changes in the number of crescents, indicating that fibrinogen deposition is an important factor in the pathogenesis of crescentic HSN. Fibrinolytic treatment can help to ameliorate the progression of renal injury and histopathological changes related to severe fibrinogen deposition in older children with crescentic HSN, although fibrinolytic treatment was not used in our study.

"Further studies should be undertaken to investigate whether fibrinogen deposition influences the clinical outcome of Henoch-Schönlein nephritis and can be used as a prognostic factor" $^{\prime \prime}$

However, there are some limitations to our study. (1) There is no clear evidence that the deposition of fibrinogen alone is responsible for crescent formation in HSN. (2) We could show no differences between the two groups in clinical outcome at the end of the follow up period, including proteinuria, creatinine clearance, and progression to chronic renal failure, and the range of intensity of fibrinogen

\section{Take home messages}

- We compared two groups of children with HenochSchönlein nephritis (HSN) treated with immunosuppressants: those with no or decreased deposition of fibrinogen (group I) and those with persistent or increased deposition (group II) after treatment

- The post-treatment activity index was significantly decreased in group I and unchanged in group II

- Age greater than 9 years at presentation was the only factor significantly related to persistent or increased fibrinogen deposition and the intensity of fibrinogen deposition at the second biopsy correlated positively with the age at onset and changes in the percentage of crescents correlated positively with the intensity of fibrinogen deposition at the second biopsy

- Thus, fibrinogen deposition plays an important role in renal injury in crescentic HSN and reflects persistent severe histological activity 
deposition was relatively limited, probably as a result of the aggressive immunosuppressive treatment. Therefore, it appears that the amount of fibrinogen deposition has no effect on clinical outcome in immunosuppressant treated patients, but is associated with persistently increased histological activity. (3) Considering the spontaneous improvement of renal symptoms in most patients with Henoch-Schönlein purpura and a possible risk of bleeding, only a small number of patients would require fibrinolytic treatment.

Nevertheless, our study suggests that persistent or increased fibrinogen deposition during the course of the disease is closely associated with severe renal injury and persistent histological activity in children with crescentic HSN. Fibrinolytic treatment should be added according to the characteristics of the individual patient and restricted to older children with the risk of severe fibrinogen deposition. Further studies should be undertaken to investigate whether fibrinogen deposition influences the clinical outcome of HSN and can be used as a prognostic factor.

\section{Authors' affiliations}

J I Shin, J M Park, Y H Shin, J S Lee, The Institute of Kidney Disease, Department of Pediatrics, Yonsei University College of Medicine, Seoul, Korea

H J Jeong, The Institute of Kidney Disease, Department of Pathology, Yonsei University College of Medicine, Seoul, Korea

\section{REFERENCES}

1 Goldstein AR, White RHR, Akuse R, et al. Long-term follow-up of childhood Henoch-Schönlein nephritis. Lancet 1992;339:280-2.

2 Counahan R, Winterborn MH, White RHR, et al. Prognosis of HenochSchönlein nephritis in children. BMJ 1977;2:11-14

3 Meadow SR, Glasgow EF, White RHR, et al. Schönlein-Henoch nephritis. Q J Med 1972;41:241-58.

4 Niaudet P, Habib R. Methylprednisolone pulse therapy in the treatment of severe forms of Schönlein-Henoch nephritis. Pediatr Nephrol 1998; 12:238-43.

5 Foster BJ, Bernard C, Drummond KN, et al. Effective therapy for severe Henoch-Schönlein purpura nephritis with prednisone and azathioprine: a clinical and histopathologic study. J Pediatr 2000;136:370-5.
6 Ronkainen J, Autio-Harmainen H, Nuutinen M. Cyclosporin A for the treatment of severe Henoch-Schönlein glomerulonephritis. Pediatr Nephrol 2003; 18: 1138-42.

7 Watanabe T, Takahashi S, Nakajo S, et al. Pathological improvement of $\lg \mathrm{A}$ nephropathy and Henoch-Schönlein purpura nephritis with urokinase therapy. Acta Paediatr Jpn 1996;38:622-8.

8 Kawasaki Y, Suzuki J, Nozawa R, et al. Efficacy of methylprednisolone and urokinase pulse therapy for severe Henoch-Schönlein nephritis. Pediatrics 2003;111:785-9.

9 Kawasaki Y, Suzuki J, Suzuki H. Efficacy of methylprednisolone and urokinase pulse therapy combined with or without cyclophosphamide in severe Henoch-Schoenlein nephritis: a clinical and histopathological study. Nephrol Dial Transplant 2004;19:858-64.

10 Morley AR, Wheeler J. Cell proliferation within Bowman's capsule in mice. J Pathol 1985; 145:315-27.

11 Neale TJ, Tipping PG, Carson SD, et al. Participation of cell-mediated immunity in deposition of fibrin in glomerulonephritis. Lancet 1988;2:421-4.

12 Bergstein JM. Glomerular fibrin deposition and removal. Pediatr Nephrol 1990;4:78-87.

13 Holdsworth SR, Tipping PG. Macrophage-induced glomerular fibrin deposition in experimental glomerulonephritis in the rabbit. J Clin Invest 1985;76:1367-74

14 Naish P, Penn GB, Evans DJ, et al. The effect of defibrination on nephrotoxic serum nephritis in rabbits. Clin Sci 1972;42:643-6.

15 Tipping PG, Thomson NM, Holdsworth SR. A comparison of fibrinolytic and defibrinating agents in established experimental glomerulonephritis. $\mathrm{Br} J$ Exp Pathol 1986;67:481-91.

16 Drew AF, Tucker HL, Liu H, et al. Crescentic glomerulonephritis is diminished in fibrinogen-deficient mice. Am J Physiol Renal Physio 2001;281:F1157-63.

17 Kitching AR, Kong YZ, Huang XR, et al. Plasminogen activator inhibitor-1 is a significant determinant of renal injury in experimental crescentic glomerulonephritis. J Am Soc Nephrol 2003;14:1487-95.

18 Miura M, Tomino Y, Yagame $M$, et al. Immunofluorescent studies on $\alpha 2$ plasmin inhibitor ( $\alpha 2$-PI) in glomeruli from patients with lgA nephropathy. Clin Exp Immunol 1985;62:380-6.

19 Ono T, Muso E, Suyama K, et al. Intraglomerular deposition of cross-linked fibrin in $\lg \mathrm{A}$ nephropathy and Henoch-Schönlein purpura nephritis. Nephron 1996;74:522-8.

20 Takemura T, Yoshioka K, Akano N, et al. Glomerular deposition of crosslinked fibrin in human kidney diseases. Kidney Int 1987;32:102-11.

21 Miura $M$, Endoh M, Nomoto Y, et al. Long-term effect of urokinase therapy in IgA nephropathy. Clin Nephrol 1989;32:209-16.

22 Davin J-C, Berge IJ, Weening JJ. What is the difference between IgA nephropathy and Henoch-Schönlein purpura nephritis? Kidney Int 2001;59:823-34.

23 Tanaka H, Waga S, Yokoyama M. Age-related histologic alterations after prednisolone therapy in children with IgA nephropathy. Tohoku J Exp Med $1998 ; 185: 247-52$. 